\title{
Hybrid vascular intervention for a giant cervical carotid artery aneurysm in a clopidogrel-hyporesponsive child
}

\author{
Osman Ocal, MD, ${ }^{1}$ Mustafa Yilmaz, MD, ${ }^{2}$ Bora Peynircioglu, MD, ${ }^{1}$ Burcak Bilginer, MD, ${ }^{3}$ \\ Ahmet Peker, MD, ${ }^{1}$ and Anil Arat, MD'

\begin{abstract}
Departments of ${ }^{1}$ Radiology, ${ }^{2}$ Cardiovascular Surgery, and ${ }^{3}$ Neurosurgery, Faculty of Medicine, Hacettepe University, Ankara,
\end{abstract} \\ Turkey
}

\begin{abstract}
Aneurysms of the extracranial internal carotid artery (ICA) in pediatric patients are rare and mostly treated surgically. The authors present the case of a child with a giant extracranial ICA aneurysm presenting with gradual airway compression. A plan was made to explore the aneurysm surgically and straighten the associated carotid artery kinks during the operation to enable further endovascular treatment if primary reconstruction of the carotid artery proved not to be possible. Finally, the aneurysm was successfully treated with staged hybrid intervention, which consisted of surgical correction of parent artery kinks followed by endovascular implantation of stent grafts. The child tolerated the procedure well without adverse events. A craniocervical CT angiogram at 5 months showed patency of the stent construct and relief of airway compression. However, a follow-up CT angiogram at 14 months showed total occlusion of the ipsilateral carotid artery without clinical sequelae and complete cessation of airway compression. The management of this was unique since the patient is the first reported case of clopidogrel hyporesponsiveness in a child undergoing neurovascular intervention with prasugrel monotherapy. Point-of-care testing-based adjustment of prasugrel necessitated education of the patient's family regarding the loss of effect of the prasugrel tablets upon crushing and contact with air.
\end{abstract}

https://thejns.org/doi/abs/10.3171/2018.3.PEDS17669

KEYWORDS hybrid; aneurysm; child; clopidogrel; prasugrel; P2Y12; vascular disorders

$\Lambda$ $\mathrm{N}$ isolated true aneurysm of the internal carotid artery (ICA) in the pediatric population is extremely rare, with only 10 reported cases in the literature. ${ }^{1,4,6,8-11,14,15,21}$ Since there is a risk of cerebral thromboembolism, mass effect, or rarely rupture, treatment is often indicated. ${ }^{23}$ In some cases, due to accompanying kinks or loops of the carotid artery, endovascular treatment may be very difficult. With only one exception, all of the reported pediatric cases were treated surgically. To our knowledge, the present case report is the first cervical ICA aneurysm treated with a staged approach consisting of an initial surgical shortening of the ICA to reduce the cervical loop, followed 4 weeks later by an endovascular implantation of a stent graft. It is also the first report of a hybrid surgical and endovascular treatment of a cervical carotid artery aneurysm in the pediatric population.

\section{Case Report}

Presentation and Examination

A 7-year-old girl, weighing $25 \mathrm{~kg}$, presented with dysphagia and hoarseness secondary to a left extracranial ICA aneurysm. CT angiography (CTA) revealed a tortuous common carotid artery (CCA) and a large fusiform extracranial ICA aneurysm extending from the carotid bifurcation to the petrous segment. Proximally, the CCA showed two $180^{\circ}$ kinks. At the hyoid and suprahyoid levels, the aneurysm caused airway narrowing (Fig. 1).

The patient had no trauma or a history of neck infection. A workup for vasculitis and infection (including tuberculosis) was negative. Vasculitis was ruled out, and, thus, no further expensive genetic tests were utilized. A decision was made to treat the aneurysm due to progressive airway compression and dysphagia. A cerebral angiogram

ABBREVIATIONS APT = antiplatelet therapy; $C C A=$ common carotid artery; $C T A=C T$ angiography; ECA = external carotid artery; ICA = internal carotid artery; PRU = platelet reactivity units.

SUBMITTED November 25, 2017. ACCEPTED March 29, 2018.

INCLUDE WHEN CITING Published online June 22, 2018; DOI: 10.3171/2018.3.PEDS17669. 

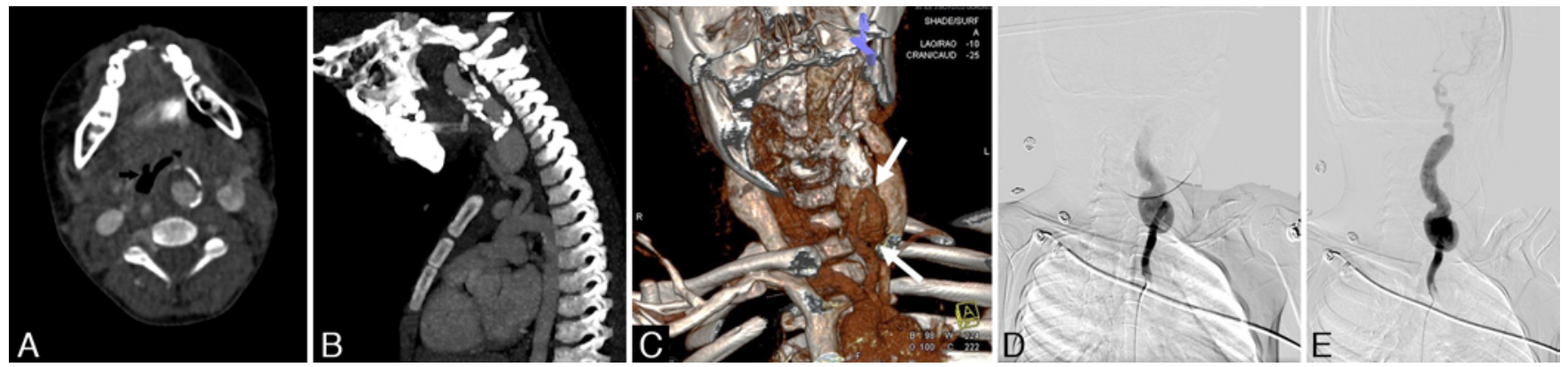

FIG. 1. Axial CTA image (A) at the level of epiglottis and sagittal maximum intensity projection CT scan (B) showing a partially clotted and calcified left ICA aneurysm extending from the common carotid bifurcation to the petrous segment and causing airway compression (black arrow, A). Volume-rendered CTA image (C) better delineating the two $180^{\circ}$ kinks of CCA (white arrows) and calcification at the aneurysm wall. Anteroposterior digital subtraction angiograms obtained during balloon test occlusion during the early (D) and delayed (E) arterial phases verifying the findings on CTA. Figure is available in color online only.

revealed that the right CCA, right ICA, and bilateral vertebral arteries were unremarkable. The giant extracranial ICA aneurysm started $6.5 \mathrm{~cm}$ distal from the left CCA origin and involved an 11-cm-long segment. Distal to the aneurysm, a $2.5-\mathrm{cm}$ normal segment was observed proximal to the carotid siphon (Fig. 1). No flow was seen in the left external carotid artery (ECA). An angiographic balloon occlusion test performed with the patient under sedation demonstrated good collateral flow to the left intracranial circulation. Due to the artery's tortuosity and kinks, endovascular treatment would have been very difficult and be associated with increased procedural risk. Similarly, an attempted resection of the lesion might have been futile due to the superior extension of the aneurysm. A resection of the mandible would be required for surgical treatment of the aneurysm as well. Consequently, we devised a plan to explore the aneurysm surgically and, if surgical treatment was likely to fail, to shorten and straighten the ICA surgically as a bailout option, enabling treatment of the aneurysm with an endovascular stent graft in a second session.

\section{Operation}

With the patient under general anesthesia, the left CCA was exposed and a huge aneurysm was observed starting from the carotid bifurcation and continuing to the petrous part of the temporal bone. The ECA was ligated and divided. The segment of the carotid artery proximal to the aneurysm was then resected, and the stump was transferred to a more proximal portion of the CCA, resulting in a shortening and straightening of this artery (Fig. 2).

Twenty-five days after the initial surgery, the patient was given a 225-mg (3-tablet) dose of clopidogrel, and platelet activity was measured by both VerifyNow (Accumetrics) and Multiplate Analyzer (Roche Diagnostics) tests, which revealed hyporesponsiveness to clopidogrel (platelet reactivity units [PRU] 320, percent inhibition 0 , adenosine diphosphate [ADP] 101, and thrombin receptor activating peptide [TRAP] 139). She was switched to prasugrel by loading her with 2 tablets of prasugrel (2 10-mg tablets, the only formulation currently available to the authors). The next day, VerifyNow and Multiplate tests showed a high level of platelet inhibition (PRU 2, percent inhibition 99, ADP 18, and TRAP 90), and a decision was made to proceed with the second stage of intervention. With the patient under general anesthesia, the right common femoral artery was cannulated with a 6-Fr $80-\mathrm{cm}$ sheath (Neuron Max 088, Stryker), and the left CCA was catheterized. The patient received a single bolus of intravenous heparin $(2500 \mathrm{U})$. A 2.8-Fr microcatheter (Cantata, Cook Medical) was navigated distal to the ICA beyond the aneurysm
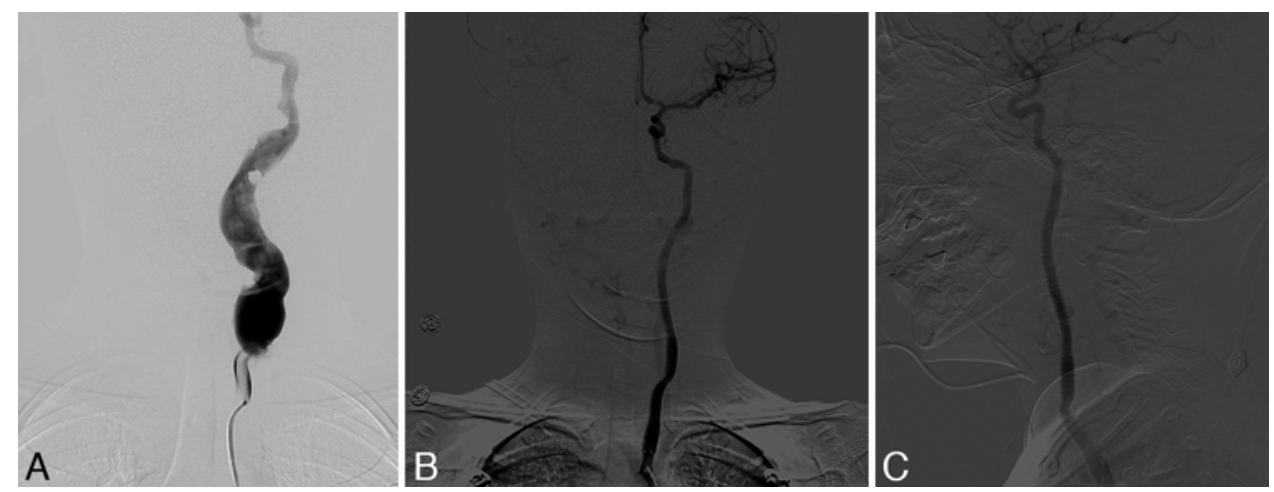

FIG. 2. Anteroposterior (AP) digital subtraction angiogram (A) showing the straightened course of the left ICA after surgical correction. AP (B) and lateral (C) carotid artery angiograms after stent graft deployment, demonstrating no evidence of a residual aneurysm and patency of the stent construct. 

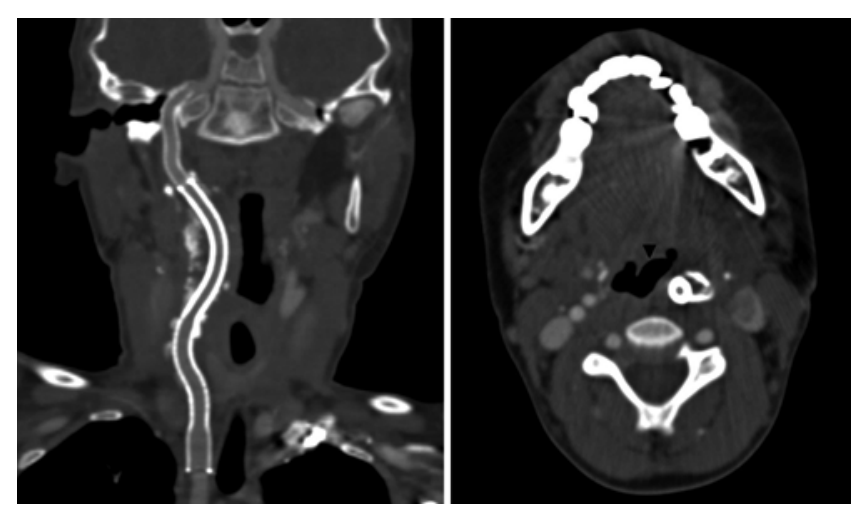

FIG. 3. Curved multiplanar reconstruction of a CTA image (left) at 5 months revealing patency of the carotid artery. Axial CTA study (right) showing resolution of the mass effect secondary to the aneurysm (arrowhead)

over 0.014-inch Transcend (Stryker) and 0.018-inch V18 (Boston Scientific) guidewires. Then a 0.058-Fr distal access catheter (AXS Catalyst, Stryker) was navigated to the cavernous segment of the ICA over the 2.8-Fr microcatheter. Finally, the sheath was navigated to the petrous segment of the ICA over the distal access catheter. A $5 \mathrm{~mm}$ $\times 10-\mathrm{cm}$ Viabahn stent graft was deployed distally, and then a second $5 \mathrm{~mm} \times 10-\mathrm{cm}$ Viabahn stent graft was deployed proximally overlapping with the first one over a short segment. An $8 \mathrm{~mm} \times 10-\mathrm{cm}$ Smart stent was deployed to ensure apposition of both stents throughout the stent construct and to prevent disconnection at the junction of the stents. A final angiogram showed no residual filling within the aneurysm (Fig. 2).

\section{Postoperative Course}

No postprocedural complications were encountered. Postoperatively, the child was started on $2.5 \mathrm{mg}$ per day (quarter of a tablet) of prasugrel. Since the pediatric use of prasugrel is not well documented, the point-of-care tests were repeated on the 2 nd postoperative day and 1 day prior to planned discharge. Unexpectedly, the results were again consistent with a hyporesponse. Upon detailed questioning of the nursing staff and the child's mother, it became evident that prasugrel tablets were divided and a quarter of the tablet was crushed in water for the child to take her prescribed dose. The child was again loaded with 2 tablets, and the family and nursing staff were educated to refrain from crushing prasugrel tablets and to administer $2.5 \mathrm{mg}$ immediately after dividing the tablet so that the drug would not lose its effect through contact with room air once the film on the tablet was destroyed by crushing. Follow-up point-of-care tests were again consistent with a relatively good response (PRU 182, percent inhibition 37).

CTA studies of the head and neck obtained at 2 days and 5 months postoperatively demonstrated patency of the stents without restenosis and serial decrease in the size of the aneurysm with cessation of the mass effect (Fig. 3). The child's symptoms had almost totally resolved as of the CTA studies obtained at 5 months, and she was kept on the same dose of prasugrel. No clinical or radiological problems were noted at the 12-month follow-up with Dop-
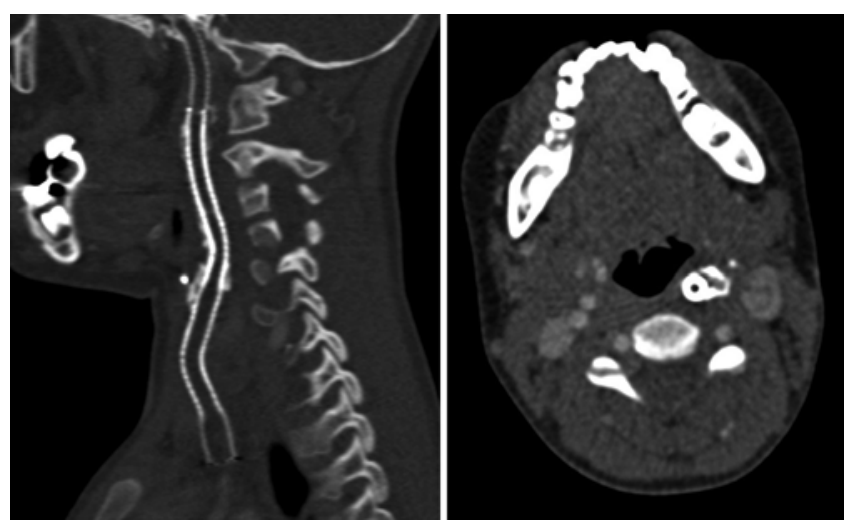

FIG. 4. Curved multiplanar reconstruction of a CTA image (left) at 14 months showing occluded stent graft and aneurysm. Axial image of the same study (right) corresponding to the level in Fig. 1A showing total resolution of airway compression.

pler ultrasound. During a follow-up telephone call placed with the mother at 14 months to schedule an 18-month follow-up visit, the mother noted that her child became reluctant to take prasugrel and finally had discontinued it about 2 months previously against medical advice. Since the child was doing very well and also since the cost of the drug was high, the mother did not insist on the administration of the drug. The child was called in for an immediate clinical and radiological evaluation. She was totally asymptomatic and neurologically intact. CTA showed total occlusion of the left internal carotid artery with patent anterior and posterior communicating arteries (Fig. 4). Antiplatelet therapy (APT) was not restarted, and the child was scheduled for cerebral MRI and noncontrast cerebral MR angiography 6 months later.

\section{Discussion}

Surgical treatment of distal ICA aneurysms extending above the line of Blaisdell is associated with serious complications, including temporomandibular joint syndrome, nerve damage, contamination with oral flora, malocclusion, and inappropriate bone healing. ${ }^{712,16}$

This has led most authors to consider minimally invasive treatments such as coil embolization or stent graft implantation for distal cervical ICA aneurysms.

An endovascular approach alone in our patient would have been extremely difficult or practically impossible due to the kinks and loops of the ICA proximal to the aneurysm. Tracking of a stent graft over the wire frequently results in obliterative elongation or spasm even if the distal part of the ICA has been cannulated with a guide wire. Since the aneurysm extended to the petrous bone, a resection of the mandible would have been required for surgery, adding to the morbidity of the procedure. Thus, we decided to proceed with the bailout plan of shortening and straightening of the ICA in preparation for a second (endovascular) procedure.

Another endovascular option would have been parent artery occlusion upon failure of the open surgical option. We opted for preserving the artery, if we could, considering the long life expectancy of the child, and we would 
possibly have reached our goal had the mother continued the antiaggregation therapy as prescribed. Finally, the data in the literature regarding flow diversion of aneurysms suggest that delayed stenosis or delayed occlusion of intracranial stents may be associated with induction of distal collateral formation, and when this happens, the patients remain asymptomatic. ${ }^{17,22}$ Nevertheless, parent artery sacrifice is a valid treatment option that would have precluded the well-defined albeit relatively small risk of our combined approach.

Three adult cases of carotid artery aneurysm treatment by a single-stage hybrid approach have been reported in the literature. ${ }^{20,24,25}$ APT was started after the procedure in 2 of these patients and during the procedure in 1 patient. The disadvantage of a single-stage procedure is that APT, which is mandatory for endovascular treatment, needs to be administered concomitantly with surgical treatment. This constitutes a disadvantage for surgery since it may increase hemorrhage-related complications. Besides, the patient may be an under-responder, resulting in an insufficient level of antiaggregation and in thromboembolic complications. The problem of surgical treatment under APT is avoided in the staged approach.

Another unique feature of the current case is that, to our knowledge, she is the first documented pediatric hyporesponder to clopidogrel subjected to an endovascular neurointervention and the first reported child undergoing endovascular intervention with prasugrel. Ticagrelor is another formula that is available in our country at a higher cost. This medication has not been studied in the pediatric population, and cost was the main reason why the patient's parent discontinued APT; this is why we opted not to start the child on this medication instead of prasugrel.

The utility of platelet reactivity tests such as VerifyNow and Multiplate in endovascular neurosurgery has been well documented in the adult population. ${ }^{13}$ Some authors reported lower rates of thromboembolic and hemorrhagic complications by VerifyNow-based adjustment of dual APT in adults. ${ }^{19}$ Recently, utilization of these tests in children has been reported. ${ }^{3,5}$ Since the stent construct became occluded asymptomatically soon after the cessation of APT, the critical role of APT in the maintenance of stent patency is clearly evident. Occlusion occurred chronically, at a time point between 12 and 14 months after the treatment, and it is plausible that partial endothelialization of the stent construct had occurred by that time, enabling a more gradual occlusion. Covered stents are associated with delayed endothelialization by interfering with neointimal proliferation and migration of smooth-muscle cells due to no existing space between stent struts..$^{18}$ Most likely, serial checking of platelet activity in the perioperative period prevented an acute or subacute stent thrombosis that could have resulted in an embolic intracranial occlusion. Verification of antiplatelet activity after the procedure also revealed another drawback of prasugrel; that is, loss of efficacy when the tablets are broken or crushed resulting in contact with room air. As of the preparation of this manuscript, pediatric formulation of prasugrel was not available to us, necessitating utmost attention by the parents to ensure immediate ingestion of prasugrel tablets once they are crushed.
Finally, our case highlights the challenges of cerebrovascular stent utilization in children. Although the sizes of the currently available neurovascular stents, flow diverters, and endovascular access systems meet the need for pediatric interventions, ${ }^{3}$ the lack of age- or weight-based pediatric APT protocols in neurointervention, as well as problems related to compliance with enteral medications in children, limit the applicability of these devices in the pediatric population. Use of bioabsorbable stent-like devices has been reported in young patients undergoing cerebrovascular interventions, but the use of these devices in children has not been reported yet, and long-term results with these devices are unknown. ${ }^{2}$ Due to these shortcomings, stents and stent-like devices should be used cautiously in children, and we suggest that APT should be monitored closely if the devices are utilized.

\section{References}

1. Antar KA, Keiser HD, Peeva E: Relapsing arterial aneurysms in juvenile Behçet's disease. Clin Rheumatol 24:7275,2005

2. Arat A, Daglioglu E, Akmangit I, Peker A, Arsava M, Topcuoglu MA, et al: Bioresorbable vascular scaffolds in interventional neuroradiology. Clin Neuroradiol [epub ahead of print], 2017

3. Barburoglu M, Arat A: Flow diverters in the treatment of pediatric cerebrovascular diseases. AJNR Am J Neuroradiol 38:113-118, 2017

4. Cinar B, Fazlioğullari O, Goksel O: True aneurysm of extracranial internal carotid artery in a 10 -year-old. Eur J Vasc Endovasc Surg 32:386-388, 2006

5. Cobb MIH, Zomorodi AR, Hauck EF, Smith TP, Fernando Gonzalez L: Optimal pediatric dosing of anti-platelet agents for pipeline stent embolization-a case report and review of the literature. Childs Nerv Syst 33:685-690, 2017

6. Edwards J, Carroll M, Wooster M, Shames M: True extracranial carotid artery aneurysm in a child. J Vasc Surg Cases 1:110-112, 2015

7. El-Sabrout R, Cooley DA: Extracranial carotid artery aneurysms: Texas Heart Institute experience. J Vasc Surg 31:702-712, 2000

8. Evans CE: Internal carotid artery aneurysm: a singular anomaly. Head Neck Surg 6:1043-1050, 1984

9. Hazarika P, Sahota JS, Nayak DR, George S: Congenital internal carotid artery aneurysm. Int J Pediatr Otorhinolaryngol 28:63-68, 1993

10. James AL, O'Malley S, Milford CA: Extracranial internal carotid artery aneurysm in a child: a diagnostic and surgical challenge. J Laryngol Otol 113:373-375, 1999

11. Kiliç E, Alanay Y, Utine E, Ozgen-Mocan B, Robinson PN, Boduroğlu K: Arterial tortuosity and aneurysm in a case of Loeys-Dietz syndrome type IB with a mutation p.R537P in the TGFBR2 gene. Turk J Pediatr 54:198-202, 2012

12. Larsen PE, Smead WL: Vertical ramus osteotomy for improved exposure of the distal internal carotid artery: a new technique. J Vasc Surg 15:226-231, 1992

13. Lee DH, Arat A, Morsi H, Shaltoni H, Harris JR, Mawad ME: Dual antiplatelet therapy monitoring for neurointerventional procedures using a point-of-care platelet function test: a single-center experience. AJNR Am J Neuroradiol 29:1389-1394, 2008

14. Lopez D, Sarac T, Lorenz R: Primary internal carotid artery aneurysm in a 15-year-old male: case report and review of the literature. Ann Vasc Surg 29:126.e1-126.e4, 2015

15. Pourhassan S, Grotemeyer D, Fokou M, Heinen W, Balzer $\mathrm{K}$, Ramp U, et al: Extracranial carotid arteries aneurysms in 
children: single-center experiences in 4 patients and review of the literature. J Pediatr Surg 42:1961-1968, 2007

16. Rosset E, Albertini JN, Magnan PE, Ede B, Thomassin JM, Branchereau A: Surgical treatment of extracranial internal carotid artery aneurysms. J Vasc Surg 31:713-723, 2000

17. Srinivasan VM, Mokin M, Duckworth EAM, Chen S, Puri A, Kan P: Tourniquet parent artery occlusion after flow diversion. J Neurointerv Surg 10:122-126, 2018

18. Takano M, Yamamoto M, Inami S, Xie Y, Murakami D, Okamatsu K, et al: Delayed endothelialization after polytetrafluoroethylene-covered stent implantation for coronary aneurysm. Circ J 73:190-193, 2009

19. Tan LA, Keigher KM, Munich SA, Moftakhar R, Lopes DK: Thromboembolic complications with Pipeline Embolization Device placement: impact of procedure time, number of stents and pre-procedure P2Y12 reaction unit (PRU) value. J Neurointerv Surg 7:217-221, 2015

20. Trinidad-Hernández M, Introcaso JH, White JV: Combined open and endovascular treatment of a saccular aneurysm and redundant loop of the internal carotid artery. J Vasc Surg 44:642-646, 2006

21. Unal OF, Hepgül KT, Turantan MI, Bozboga M, Yazicioglu E: Extracranial carotid artery aneurysm in a child misdiagnosed as a parapharyngeal abscess: a case report. J Otolaryngol 21:108-111, 1992

22. Wajnberg E, Silva TS, Johnson AK, Lopes DK: Progressive deconstruction: a novel aneurysm treatment using the pipeline embolization device for competitive flow diversion: case report. Neurosurgery 10 (Suppl 1):E161-E166, 2014

23. Wolfe SQ, Mueller-Kronast N, Aziz-Sultan MA, Zauner A, Bhatia S: Extracranial carotid artery pseudoaneurysm presenting with embolic stroke in a pediatric patient. Case report. J Neurosurg Pediatr 1:240-243, 2008

24. Wong E, Chue WL: Hybrid (open and endovascular) repair of distal extra-cranial internal carotid artery aneurysm. EJVES Extra 20:e19-e21, 2010
25. Wu J, Chen Y, Qu L, He Y, Zhi K, Bai J, et al: Using PTFE covered stent-artery anastomosis in a new hybrid operation for giant juxta-skull internal carotid aneurysm with tortuous internal carotid artery. Int J Cardiol 185:25-28, 2015

\section{Disclosures}

The authors report no conflict of interest concerning the materials or methods used in this study or the findings specified in this paper.

\section{Author Contributions}

Conception and design: Arat. Acquisition of data: Arat, Ocal. Analysis and interpretation of data: Arat, Yilmaz. Drafting the article: Ocal. Critically revising the article: Arat. Reviewed submitted version of manuscript: all authors. Approved the final version of the manuscript on behalf of all authors: Arat. Administrative/technical/material support: Arat, Yilmaz, Peynircioglu, Bilginer, Peker.

\section{Supplemental Information}

\section{Previous Presentations}

Portions of this work were presented in oral form at the 14th Congress of the World Federation of Interventional and Therapeutic Neuroradiology, Budapest, Hungary, October 18, 2017.

\section{Correspondence}

Anil Arat: School of Medicine, Hacettepe University, Ankara, Turkey.anilarat@hotmail.com. 